\title{
THE PECULIARITIES OF INCOMPLETE SENTENCES IN MODERN ENGLISH
}

\author{
${ }^{1}$ Nilufar Sadullaeva, ${ }^{2}$ Umida Burieva \\ ${ }^{1}$ Associate professor, DSc, National University of Uzbekistan, Tashkent, Uzbekistan. \\ E-mail: nilufar_sadullaeva@mail.ru \\ ${ }^{2}$ Senior teacher, National University of Uzbekistan, Tashkent, Uzbekistan. \\ E-mail: umida.burieva.1984@mail.ru
}

\section{ABSTRACT:}

The issue of incomplete sentences has been long attracted more scientists. There were no special works devoted to incomplete sentences. The main significant research of this matter were the thesis of A.N.Nazarov and the work of I.A.Popova. I.A.Popova, at the conclusion of her work, comes to the pessimistic conclusion that it is impossible to give a satisfactory definition of incomplete sentences based on a grammatical structure, as well as the impossibility of putting forward clear criteria for dividing sentences into complete and incomplete[1]. This conclusion largely depended on the fact that the definition of incompleteness for a long time was based not on structural-grammatical, but on semantic features. So A.N.Nazarov believed that semantic incompleteness allows defining incomplete sentences and their criteria. Since these conclusions were made, the issue of incomplete sentences and many other problems related to it were investigated thoroughly.

Nevertheless, many questions of the theory of incomplete sentences still remain unresolved, their structure and peculiarities, also the relationship between various linguistic factors have not been completely clarified.

Keywords: incomplete, incompleteness, ellipse, descriptive, lexical meaning, complex sentence, context, the omitted words, non-expanded replica, working definition, situational, contextual, syntactic distribution, structural-grammatical concept, subsequent sentences, secondary members of the sentence.

Article Received: 18 October 2020, Revised: 3 November 2020, Accepted: 24 December 2020

\section{Introduction}

A clarification of the concept of incomplete sentences and definition of complete sentences in general, because only through shared understanding can be built the concept and definition of incomplete sentences as a private matter of theory question in the sentence.

The definition of the sentence has caused and still causes numerous disputes and interpretations not only between linguists of different directions, but even between the ones that are in one direction, and a satisfactory solution to this question has not been found yet. Therefore, we will continue to rely on the definition given by the Academic grammar of the Russian language, which, from our point of view, most fully covers the different sides of the sentence and which we use as a "working definition": "A sentence is a grammatically formed integral unit of speech, which is the main means of forming, expressing and communicating thoughts. The sentence expresses not only messages about reality, but also the speaker's attitude to it. Each sentence from the grammatical point of view represents the internal unity of its verbally expressed members, their order of arrangement and intonation" [2].

In this article, the term "incomplete sentence" is considered as referring to two-part sentences. Thus, an incomplete sentence is a twopart sentence. It can also be part of a compound or complex sentence. 
From the point of view of message transmission, all sentences of coherent speech are, i.e. they convey what needs to be conveyed, otherwise they would not be sentences, since they could not fulfill their main, communicative function.

Each sentence, being in the context, fully conveys the idea contained in it, regardless of its structural features, because if the sentence performed the function of transmitting the message incomplete, communication between people would be impossible. And, if we consider the question from this point of view, there are no incomplete sentences at all.

Such replicas of colloquial speech, which, I.A.Popova classifies as "undeveloped" or "actually incomplete sentences", which express insufficiently differentiated thought, unresolved into distinct and in a certain way combined verbal representations, in other words, those in which the thought itself has not yet been sufficiently formed, has not yet completely turned into a phenomenon of the language. Such "offers" cannot be considered offers at all. [3]

This definition is inherited from A.A. Shakhmatov and can be categorized as "sufficient" and "insufficient" differentiated, in other words, if some thought "has not been insufficiently" formed yet, has not evolved fully yet into the phenomenon of language, if it is already made, it will never become a phenomenon of speech, i.e. will never be formed into a sentence. To some extent this was noted by the author of the article, calling such replicas "language formations that have not become full sentences yet". Thus, in this case, the term "incomplete" is out of bounds of the grammar and refers to a certain concept, in grammar, the term "incomplete proposal" describes specific positional model, that are relative to the positional models of full sentences. Therefore, the term "incomplete sentence" can not be attributed to non-expanded replicas, since these last ones, as the author himself points out, were not formed into a sentence.
Based on the evidence that were listed above, we come to the conclusion that when considering a sentence in context, from the point of view of completeness of meaning, all sentences are complete, i.e. incomplete sentences, from this point of view, do not exist at all. In a particular context or situation, each sentence, according to its structure and function, is complete in terms of content. In this regard, we cannot agree with the author of the dissertation "incomplete sentences in Russian" A.N.Nazarov, who claims that "the incompleteness of a sentence is determined primarily by the semantic incompleteness of its content" [4]. This attitude leads to the study of grammatical phenomenons, based only on the subjective interpretation of the meaning of the corresponding sentences, on the intuitive linguistic flair of the researcher, and entails purely subjective assessments and conclusions.

Methods

This work uses a descriptive research method based on the example of Z.Harris and CH.Friz. Their application has made it possible to reduce the large variety of specific sentences found in texts to a limited number of models.

In some cases, elements of transformational analysis were used to prove certain positions, which "serve to identify language units at the syntactic level", therefore, the use of these methods has proved to be successful for elucidating the identity of some models of incomplete and complete sentences.

To determine the boundaries of the distribution of a particular model of incomplete sentences, depending on the lexical meaning and grammatical form of words that are part of it, a calculation was used, which made it possible to find out a certain degree of probability of the appearance of a particular model of incomplete sentences.

\section{Discussion}

The error of the position expressed by A.I.Nazarov is also proved by the fact that, taking a sentence out of context (and in the analysis, as a rule, each sentence is considered in isolation, out 
of its connection with the context), we can hardly talk about any semantic completeness of the sentence at all. It is on this basis that I.A.Popova came to the conclusion that "if the criterion for determining incompleteness is considered to be a sentence for its semantic incompleteness, then almost all of our speech, not only oral, dialogic, but book-written, will be considered as "incomplete" sentences [5]. After all, a significant part of formally complete sentences of coherent speech, taken outside the context, does not express the completeness of thought that they possess in the context. The semantic connection of context sentences and the relative inferiority of an isolated sentence is expressed in various means: in the use of pronouns (personal and demonstrative), conjunctive particles, conjunctions at the beginning of the sentence, introductory words, and in other ways. For example, the sentences: "His political work is smaller in volume and narrower in range than that of his two great contemporaries, but it reflects more clearly than the poetry of either, the collapse of faith that was a tragedy in many sincere lives of the period"; nevertheless, it cannot be considered complete in meaning, or even clear in terms of content at all, without a preceding sentence that reveals the subject relationship of personal pronouns in this sentence: "Eminent alike as poet and critic, Matthew Arnold holds a place of singular distinction among Victorian writers"[6]. But even this last one does not bring complete clarity without a more extensive previous context, since the content of the group "two great contemporaries" remains unclear [7]. When a sentence is placed in context and the researcher considers it as a part of the whole, it acquires full meaning. This was noted by V.V.Vinogradov when he wrote: "taking into account all the means of expression, situation and context, taking into account the structural and grammatical features of the so - called incomplete sentences, almost every one of them will be "complete", i.e. adequate to its purpose and appropriately performing its communicative function"[8]. When considering the incompleteness of a sentence, we rely on the fact that the sentence is from the side of its model, and only from this side, is not the product of a creative act, but represents an already ready reproducible unit. "The positional model of the offer is a ready-made tool, a ready-made structure". A sentence, from the point of view of its model, is a unit of language, and, like any unit of language, it is It is a unit of language, not because it can express a complete thought, but because it is an established structure, a ready model that is reproduced in speech as an appearing structure. With that being said T.P.Lomtev "ready replicable unit" proposal is "only by its model, but by its function, in the act of communication, it is the result of creative activity, not reproduction".[9] Precisely, because the thought expressed in a sentence is the work of the speaker, something is always produced, something is conveyed or established in each sentence. However, the model of the sentence and its structure do not change, remaining within the framework of the positional structures that exist in the language.

At the same time, there may be errors in speech from the models that have been developed in the language system: some parts of these models may fall out, but this does not mean that such a sentence: "Wish I were the same" - is not a new construction, but represents the same positional model (structure) as the sentence "I wish I were the same". Or an offer: "You coming, Scobie?" - it can only serve as a unit of communication because its positional model is built in exact accordance with the positional model of the sentence "Are you coming, Scobie?" Such errors or incomplete structures are based and constructed on the basis of those models that are already available in the language system. These offers are only understandable because they are built in the exact accordance with the full offer. For example, a sentence like: "Very pretty, that" or: Queer being, my mother" -would hardly be clear if they were not based on the same models as in the sentence "Very Pretty that is", or "A queer 
being my mother is" in which there is an inversion of the position of the nominal part. "Very pretty that" the model is constructed in exact accordance with "Very Pretty that is" model, but it lacks one verbal form. And understanding a sentence based on an incomplete model depends on understanding the sentence reproduced in speech. This was underlined by A.M.Peshkovsky: "Incomplete sentences in colloquial speech are much more than complete ones in our consciousness, they are always equal to full ones". A.M.Peshkovsky considered this feature to be the most important [10]. Complete sentences exactly correspond to the model, the structure of the sentence in the language, in other words, they represent this model. In the context of the sentence, the positional model of the sentence of this language syntactic relations is revealed. Incomplete sentences stand out from complete ones. They represent the same model as the complete ones, but without any positional links. The presence of incomplete sentences is due to the possibility of contrasting them with full ones. If you can say: "Sounds like a good idea" or "What a nice speech!", then it is because there is a model of the complete sentence "It sounds like a good idea" or "What a curious shape it is!"'. And vice versa, since there is a model in a sentence "What a nice speech! I can't imagine why people speak against her" will be incomplete. They will not be incomplete in meaning, since they fully express the thought contained in them and are incomplete only from the point of view of their structure, i.e. the term "incomplete" can be attributed to them only from a grammatical point of view. This means that an incomplete sentence is a purely grammatical concept and has no relation to the plan of content, to the completeness of the thought of a single sentence. The incomplete sentence is a breach of positional models, which is possible due to the fact that the individual position element so closely linked that one position of the verbal form may assume a different position in the other verbal forms, and even the whole positional model of its sentence. For example:
Hallo, Jim, how is it going?

Badly.

I'll wait for you, he said in an urgent undertone.

In the porch.

Both selected sentences are correlated with complete sentences by the absence of verbal form in the predicate position, since these sentences are represented by dependent circumstantial positions that assume the presence of the predicate position, and through it, the subject: in the first case, "It is going bad, in the second, I'll wait in the porch".

Due to this mutual conditionality, or in V.G.Admoni's terminology, "projection of syntactic relations" of copula position, the internal connection indicates the loss of the verbal form in one or more elements. In addition, the loss of the verbal form in one of the sentences is also indicated by the corresponding rows of sentences. T.P.Lomtev wrote about this: "The position of the verbal form in a sentence is determined not only by the data that represent a single sentence taken separately, but also by those that represent correlative series of different types of sentences, i.e. language systems in general". Based on these evidences, like T.P.Lomtev, we believe that the difference between incomplete sentences and complete ones is not dependent on the absence of certain positions, but in the fact that "they are presented negatively, i.e. they are not represented by separate verbal forms" [11]. This means that the verbal form may not be represented in any position, but still the position itself exists. For example, in a sentence:

"Should not think you'll last five minutes without me to explain the score to you"- there is no verbal form in the subject position, but the position itself exists and determines the predicate position. Or in the sentence: "Sensible woman, Lady Windermere" -there is no verbal form in the copula position, but it defines the relation of the position of the nominal part to the position of the subject.

Thus, an incomplete sentence is a sentence whose positional model is not fully expressed in 
verbal forms, in which one or more positions are expressed negatively. This is confirmed by the bundles and forms in the existence of copula positions in a sentence. Incomplete sentences are correlated with complete sentences by the presence or absence of a verbal form in a particular position. They exist in speech along with complete sentences and convey the content of the message due to two factors, indicated by A.M.Peshkovsky, particularly, due to the context and the situation of the speech (situation).

Context, consequently, the situation (or common context), because their functions are identical, plays an important role for the transmission and content of not only the complete sentence, but even the meaning of a single word ( in the case of its polysemy). By the context, we mean the set of speech conditions in which a given sentence is used. Our understanding of the context is based on the definition given by V.G.Kolshansky: "From the linguistic point of view, the context can be defined as a set of formally fixed conditions under which the content of a language unit is clearly identified. At the same time, unambiguity should be understood as the manifestation of only one specific content of the language form under given conditions" [12]. Context is a speech factor that creates a condition for the use of incomplete sentences by the material composition of their words and forms.

In incomplete sentences, context and situation play a very special and pivotal role. They create conditions for the formation of incomplete sentences and make it possible to use them. Here, the context and situations not only specify and clarify the content of the sentence, but, most importantly, make it possible not to name any objects or concepts due to the fact that they have already been named in previous sentences, or due to the fact that the situation of speech or previous experience of the speaker makes the content of missing verbal forms understandable to the companion:

"Old Miss Mc Glome always sings at this hour. But not tonight. Songs of her girlhood, she says. Hard to think of her as a girl. Wonderful women though. Connaught. Thirty-nine today, sound as bell, apart from my old weakness, and intellectually I have now every reason to suspect at the crest of the wave-or thereabouts.

Very softly. Go very softly.

The difference between context and situation is that the context is a speech factor, and the situation is an extra speech factor; therefore, in a situation, a verbal form that is not named in an incomplete sentence does not receive any verbal form at all.

It is about such proposals that A.M.Peshkovsky wrote that "there is no omission, no omission, no deliberate omission of words... In all such cases, the speaker does not imagine the omitted words, does not remember them because they do not need them" [13].

Rycker's voice said, "He laughed at me. How dare he laugh at me?" The doctor said to Brother Philippe, "I have his head. Can you find his legs? We've got to get him inside". He called to Rycker, "Put down that gun, you fool, and help!"

"Not at Rycker", Querry said. The doctor leant down closer: he could hardly hear him. He said, "Don't speak. We are going to lift you now. You'll be all right".

Querry said, "Laughing at myself "(G.Greene)

However, these differences between context and situation do not matter when we talk about the structural features of incomplete sentences, and do not entail changes in the types of "incompleteness" of the sentence. The context and the situation, as N.N.Amosova notes, "can act in one direction" [14]

\section{There is no verbal form in the subject position.}

There are no verbal forms in the subject and predicate positions.

"What did you say? In the evening told Louise that

He touched her I should have to retire before end of tour". 


\section{There are no verbal forms in the subject and predicate positions.}

As can be seen from the examples given "when borrowing words from the surrounding speech" (from contexts), and in the case of "replacing them with real representations from the speech environment", the structure of incomplete sentences, their types remain the same. Therefore, in both cases we are dealing with the same positional model of the sentence. And since the model of an incomplete sentence is not subject to any changes, since the typology of the sentence is the same, then, in our view, it is necessary to divide incomplete sentences into "situational" and "contextual", as I.A.Popova does, this classification characterizes incomplete sentences in terms of external, visual, rather than internal, structural factors [15].

Moreover, in book-literary speech, both these factors (context and situation) are intertwined so closely that it is sometimes impossible to determine which of the factors causes the incompleteness of the sentence in this particular case.

"Hillary: You subscribe to the Racquets Club, and worked your way through college. Or did you get a football scholarship?

Charles: Correct". (Situation).

As it can be seen from the examples given, the context and situation are intertwined so closely that it is difficult to determine which factor in each particular case causes the possibility of using an incomplete sentence, in some cases one of them compensates for some insufficiency of the other. This distinction is not necessary for analyzing the structure of each separate sentence, since this division does not reflect the structural features of the sentences under consideration. Therefore, the context and situation, are important factors that determine the possibility of using incomplete sentences for situational and contextual, is not justified by the facts of the language [16].

The impact of context as a condition for the appearance of an incomplete sentence may be different. There are three types of context to distinguish between:

1) prepositive,

2) interpositive

3) postposeitive.

The most common type of context as a condition for the appearance of an incomplete sentence is the prepositive context. By prepositive context, we mean the verbal expression of a word form omitted in an incomplete sentence before the appearance of the most incomplete sentence, in one of the preceding sentences [17]. In a prepositive context, there may be 1) the same word form that is then omitted in an incomplete sentence, or 2) the word form that is then omitted in an incomplete sentence:

In the second case, the incomplete sentence omits the pronoun in the subject position, indicating the person or object named in the previous sentence:

"A very nice mail, I said. Tells you're not forgotten".

An interpositive context is also widely used as a condition for incomplete sentences. By an interpositive context, we mean those verbal forms that are represented in the most incomplete sentence. This can be either 1) verbal expression omitted word forms in the sentence, or 2) the presence of others shapes, valence for the omitted word form, or 3) the presence of incomplete sentences, synthetical distribution, which indicates what kind of word is omitted.

1. In the first case, we are always dealing with some variant of the omitted word form, acting in one of the secondary positions:

"Broke a little bone in her ankle, doctor said".

"Know my way".

"Think you can tell me what to do, a damned kid like you".

2. The second type of interpositive context occurs only in the case of omission of the 3rd person pronoun. Numbers in the subject position if there is a predicate verb in the Present Indefinite position. Sometimes the verb form ending on $-\mathrm{s}$, 
valence on the word form of the 3rd person pronoun. The number in the subject position is the interpositive context that can be considered a formal factor indicating the ambiguity of the omitted word form in the subject position [18].

"Sooner you go to the better. Makes no odds to me".

"I'm ready when you're, Fred said. Suits me".

But more often both these types of interpositive context are combined, especially in cases when the lexical meaning of the verb in Present Indefinite in the predicate position cannot serve as a formal sign for choosing the 3rd person pronoun.

"Likes his trouble up to the neck, eh?".

"Takes after her daddy -don't you, duck?"

3. In some cases, the incomplete sentence itself contains such verbal forms, the syntactic distribution of which indicates the omission of certain word forms in the positions of the subject and predicate.

"Nice of you to say so, sir".

The syntactic distribution of the adjective "nice+infinitive" indicates an ellipse of the word forms "it" and "is" in the main positions. Also in offers:

"A pity you got the sack, I put in".

"First time I'd ever had it".

The syntactic distribution of the "pity" and "time" indicates the lexical meaning of the omitted word forms.

In a postpositive context, the word form omitted in an incomplete sentence is expressed in subsequent sentences. In this case, it is usually called the non-variant form of the word form omitted in the incomplete sentence and in the same position as in the incomplete sentence.

"-Pulled down all my lights without even asking.

-But they'll pay for it".

Can't hear you, old man, not sausage. Up a

bit.

Give. I still can't hear you.

Mustn't overdo it.
Straight up. It's straight up, son.

Not so wonderful for a kid of four-and it wasn't because you were wonderful either".

In most cases, none of these types of context is the only factor that creates conditions for incomplete sentences. Usually, the condition for incomplete sentences is created by the interaction of several types of context.

"-It makes you laugh.

-Didn't make me laugh. It made me think".

The incomplete sentence "Didn't make me laugh" appeared as a result of the presence of a prepositive context (the form "It" in the subject position in the previous sentence), supported by a postpositive context ("It" in the subject position in the following sentence).

"She's got her tricks to hide it. Smiles and looks nice. When she hasn't heard what you said". (Prepositive and interpositive context). Since basically the set of conditions for creating incompleteness is manifested not in one sentence, but in a number of consecutive or sometimes even separated sentences, it is correct to assume that the set of speech conditions that create the possibility of using incomplete sentences is combined by a macro-context [19].

Having come to the conclusion that incomplete sentences are a structural and grammatical concept, and not a semantic one, and taking this position as a basis, it is necessary to decide in such positions that the absence of a verbal form entails the incompleteness of the sentence. Does this apply to the main positions or also to secondary ones? In order to answer this question, we must first consider the structure of a complete two-part sentence and find out the significance of each position".

The main positions of a two-part sentence -the subject position and the predicate positionrepresent the basis around which the sentence is built. This is the structural center of the sentence, from which all other positions are depended on. This position is well proved and illustrated by the analysis of any two-part complete sentence on the components themselves, as a result of which we 
always come to the simplest formula. L.Bloomfield calls the "action-action" model the norm of the sentence ("Favorite sentence - form") [20].

The fact that it is the position of the subject and predicate that form the basis of the structure of a two-part sentence, also proves by the fact that, they can already form a complete structure by themselves, for example, "John ran".

In common sentences, they are the centers around which other positions are grouped: The "main positions are" determinative and independent.

But the main positions are only the structural center. In terms of content, they often do not bear the main burden. The semantic center of a sentence may lie in the secondary positions:

"From the river came the summons of the ship's bell". next".

"Impatiently the doctor called out the

In all these sentences, the content of the message is conveyed not by the verbal forms that appear in the main positions, but by the verbal forms that appear in secondary positions. If only the main positions are taken in these offers:

1) came the summons, 2) Doctor Colin had known, 3) the doctor called out, their content will not be only closely joined together, but even (in the second and third cases) almost loses its meaning. On the other hand, if you remove these verbal forms, the sentence immediately splits into unrelated separate words and phrases: "From the river...of the ship's bell, Impatiently...the next number" which, taken together, do not express anything in terms of the message at all. It's a set of words, and that's all. The sentence fell apart, because despite the fact that all the verbal forms that carry the main content of the message remained, what defines the structure and creates it, has disappeared. We did not only remove the verbal forms in the main position, but also destroyed the positions themselves, i.e., destroyed the structure. An inequality is created: the structural center is the center of the message. Therefore, in the absence of verbal forms in the position of additional information, definitions, and circumstances, the sentence structure is not destroyed, but the message content partially or completely disappears. On the other hand, by destroying the positions of the main members in the sentence, we destroy the structure of the sentence, because, as A.G.Rudnev underlined in his article "secondary members of the sentence cannot exist without explicit or implicit organizing centers (subject or predicate)" [21].

Considering all the evidence that we talked about before, we come to the conclusion that incomplete sentences can be considered only those that do not have a verbal form in one of the main positions or in both together, since we consider incomplete sentences only in terms of structure and grammar, and not in terms of meaning, because the latter in this particular case, from our point of view, takes us beyond the field of grammar.

In English, incomplete sentences are common, in which there are no verbal forms in the position of the subject and predicate (or part of the predicate), but secondary positions are verbally expressed:

"Am I nuisance?-Never".

"Hallo, Jim, how is it going?-Badly".

"You must have had plenty of practice, surely.-Not much, as matter of fact".

Such sentences exist and can only exist because the positions of the main embers remain in them, and are not violated, even though verbal form is absent. Thus, the structure of the sentence is preserved, although, only what is new in terms of the message is expressed verbally [22].

Since "secondary positions are not determinative and independent", they do not organize the structure. The analysis of incomplete offers should only be based on studying the structural features of this type of offer. And with this approach, it is clear that the absence of secondary positions does not create incompleteness of the sentence, and only such 
sentences can be considered incomplete if one or both of the main positions are represented negatively (not represented by verbal forms).

Conclusion

To sum up, we come to the conclusion that an incomplete sentence is a simple two - part sentence, the positional model of which is not fully expressed in verbal forms, in which one or both of the main positions are represented negatively. This is confirmed by the connections and forms of available copula position. Incomplete sentences relate to the complete sentences and convey the content of the blogger's message by two factors: context and situation. The absence of word forms in secondary positions does not create incompleteness of the sentence. The concept of "incomplete sentence" is a structural and grammatical concept, not a semantic one.

References

[1] Admoni V.G. Completeness of construction as a phenomenon of syntactic form. Voprosy yazykoznania, №1, 1958. P.111-117

[2] Barsova O.M. Two-part verbal sentences in English. NDVSh FN, 1962.

[3] Burieva U.A. "Characteristic features of incomplete sentences". Theoritical and applied sciences

Volume:80/Issue:12/December/2019, P.557-560.

[4] Burieva U.A. "Incomplete syntactic constructions in cognitive-discoursive information and isofunctional aspects". IJRD Volume:5/Issue:4/April 2020, P.344.

[5] Chomsky N. Syntactic structures. New In Linguistics, vol. 2, -M., 1962.

[6] Dudyk P.S. Incomplete and elliptic sentences in the modern Ukrainian language. abstract of cand...diss. -Kiev, 1954.

[7] Grammar of the Russian language. Academy of Sciences of the USSR. -M., 1954.
[8] Harris Z. Joint occurrence and transformation in the linguistic structure. "New in Linguistics", vol. 2, -M., 1962;

[9] Kolshansky G.V. On the nature of the context. -NY. 1959.

[10] Fries Ch. The structure of English. -N.Y., 1952.

[11] Fitialova S.Ya. Problems in structural linguistics. -M., 1962. p.108.

[12] Lomteva T.P. Fundamentals of the syntax of the modern Russian language. Uchpedgiz, 1958.

[13] Nazarov A.N. Incomplete proposals, conditions for their use and their structure. -Penza, 1950.

[14] Popova I.A. Incomplete sentences in modern Russian. Proceedings of the Institute of Linguistics, vol. 2, -M., 1953.

[15] Peshkovsky A.M. Ruskiy sintaksis v nauchnom osveshenii. -M. Uchpedgiz. 1956. - 511p.

[16] Shvedova N.Y. Typology of monolithic sentences based on nature and paradigms/Problems of Modern Philology. -M., 1965.

[17] Sampson G. The Concise Cambridge History of English Literature. Cambridge University Press. -Cambridge. 2002

[18] Sadullaeva N.A., Mamatova F.B. Classification of euphemism and its formation in the Uzbek language. Journal of critical reviews. Vol 7, Issue 4. (Scopus) March 23, 2020. Malaysia. ISSN 2394-5125

http://www.jcreview.com/fulltext/1971584972123.pdf/1586606700

[19] Sadullaeva N.A., Arustamyan Y.Y., Siddiqova I.A. Assessment of educational process and its organization. International Journal of Psychosocial Rehabilitation, Vol. 24, (Scopus). Special Issue 1, 2020. ISSN: $1475-719$ https://www.psychosocial.com/article/PR2 01193/11922/ 
[20] Sadullaeva Sh.A., Arustamyan Y.Y.,

Sadullaeva N.A. Shifting the Assessment

Paradigm from Knowledge to Skills:

Implementation of New Appraisal

Procedures in EFL Classes in Uzbekistan.

International Journal of Innovative

Technology and Exploring Engineering

(Scopus) 2019, Индия

https://www.ijitee.org/wp-

content/uploads/papers/v9i1/A9179119119

.pdf

[21] Toporov V.N. On the boundaries of the application of the transformation method/Abstracts at the conference on structural linguistics dedicated to the problems of the transformation method. M., 1961.

[22] Vinogradov V.V. Basic questions of the syntax of a sentence (on the material of the Russian language). Academy of Sciences. -M., 1955. 